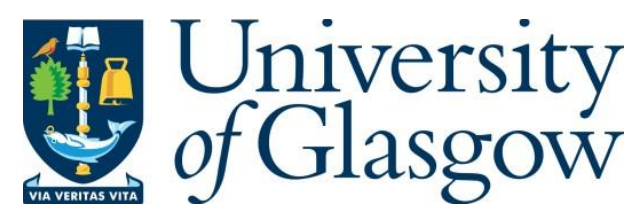

Loupy, G.J.M. and Barakos, G.N. (2017) Processing and analysis methods for transonic cavity flow. Physics of Fluids, 29(7), 076105.

There may be differences between this version and the published version. You are advised to consult the publisher's version if you wish to cite from it.

http://eprints.gla.ac.uk/143870/

Deposited on: 10 July 2017

Enlighten - Research publications by members of the University of Glasgow http://eprints.gla.ac.uk 


\title{
Processing and Analysis Methods for Transonic Cavity Flow
}

\author{
G.J.M. Loupy ${ }^{1}$, G.N. Barakos ${ }^{2}$ \\ CFD Laboratory, University of Glasgow, Glasgow, G12 8QQ, UK
}

\begin{abstract}
This paper focuses on the localisation of noise sources in transonic cavity flows. Beamforming is used to estimate the pressure fluctuations inside a resonant transonic cavity, showing the localisation of the main sources of noise using an acoustic array and also combining it with a mean flow-field. The influence of the microphone array position, density, and shape are investigated. The presented method models the noise propagation with simple assumptions that are easily applicable to wind tunnel testing, and may help localise the noise sources from complex geometries without intrusive methods.
\end{abstract}

\footnotetext{
${ }^{1} \mathrm{PhD}$ Student. g.loupy.1@research.gla.ac.uk

${ }^{2}$ Professor, MAIAA, MRAeS, MAHS. george.barakos@glasgow.ac.uk, corresponding author
} 


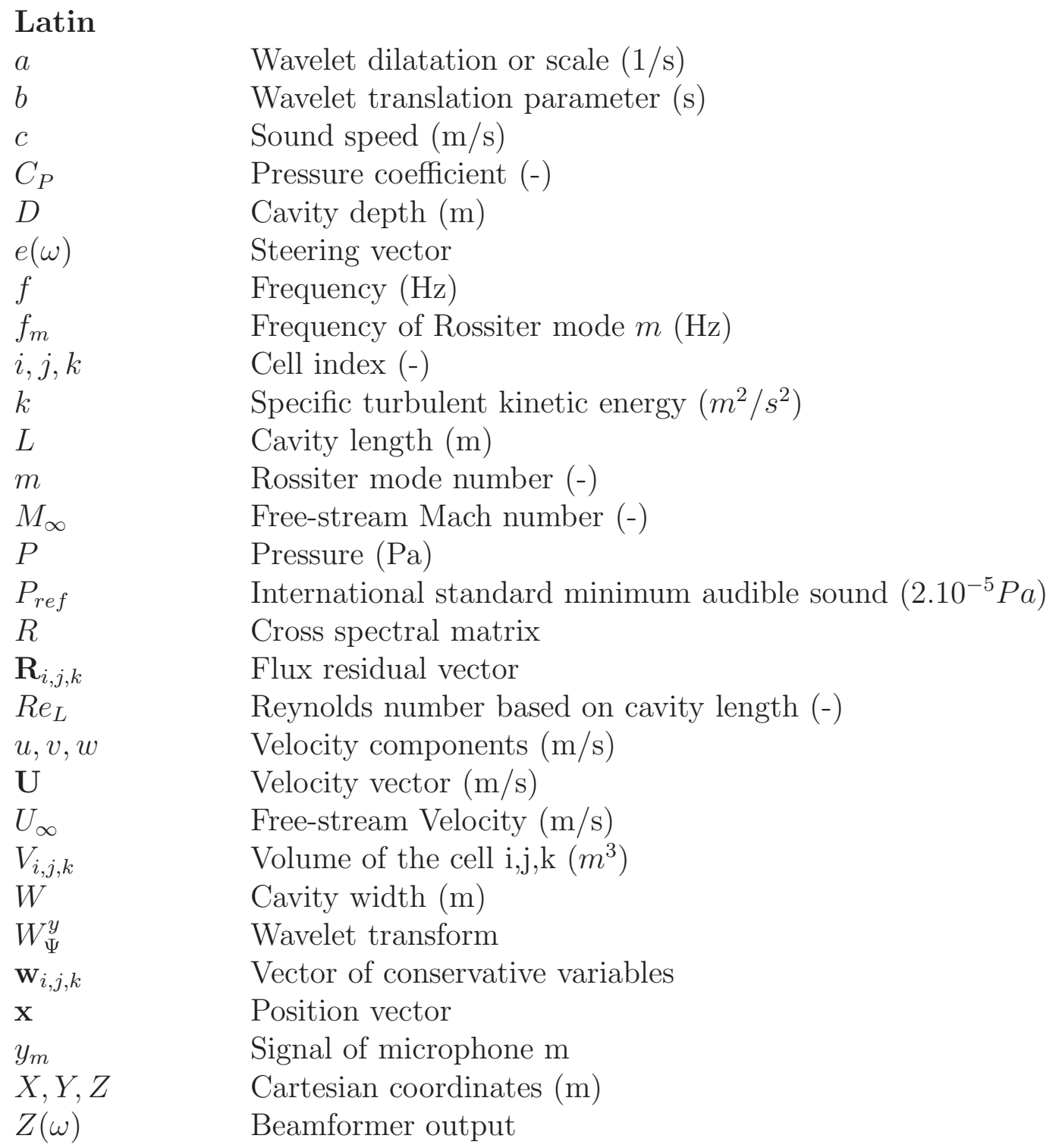




\section{Greek}

$\alpha$

$\Delta f_{\text {ref }}$

$\Delta m$

$\kappa_{\nu}$

$\gamma$

$\omega$

$\omega_{0}$

$\Psi(t)$

$\rho$

\section{Acronyms}

$A L E$

$B I L U$

BISPL

$B I W$

$C F D$

$C F L$

FFT

$H M B$

$M E M$

$M U S C L$

$M U S I C$

$O A S P L$

PIV

$P S D$

RK4

$S A S$

$S P L$

$U C A V$

URANS
Rossiter Phase shift (-)

Reference frequency $(\mathrm{Hz})$

Beamforming time delay ( $\mathrm{s}$ )

Rossiter convection velocity coefficient (-)

Ratio of specific heats of air (-)

Specific turbulence dissipation rate $(1 / \mathrm{s})$

Pulsation $(2 \pi f)(1 / \mathrm{s})$

Mother wavelet (-)

Density $\left(\mathrm{kg} / \mathrm{m}^{3}\right)$

Arbitrary Lagrangian-Eulerian

Block Incomplete Lower-Upper

Banded Integrated Sound Pressure Level

Banded Integrated Wavelet

Computational Fluid Dynamics

CourantFriedrichsLewy

Fast Fourier Transforms

Helicopter Multi-Block

Maximum Entropy Method

Monotone Upwind Schemes for Scalar Conservation Laws

MUltiple SIgnal Classification

Overall Sound-Pressure Level

Particle Image Velocimetry

Power Spectral Density

Runge-Kutta method 4th order

Scale Adaptive Simulation

Sound-Pressure Level

Unmanned Combat Air Vehicle

Unsteady Reynolds-Averaged Navier-Stokes 


\section{Introduction}

Transonic cavity flows have strong acoustics including broadband noise and tones, called Rossiter modes [1], generated by a complex interaction between the shear layer spanning the opening, and reflected acoustic waves inside the cavity [2]. Rossiter's theoretical model gives the modal frequencies, based on the interaction between an assumed periodic shear layer vortex shedding, and the acoustic waves travelling along the bay. The semi-empirical formula available for the estimation of the tonal frequencies, as modified by Heller [3] is:

$$
f_{m}=\frac{U_{\infty}}{L}\left[\frac{m-\alpha}{M_{\infty}\left(1+\left(\frac{\gamma-1}{2}\right) M_{\infty}^{2}\right)^{-1 / 2}+1 / \kappa_{\nu}}\right]
$$

where $f_{m}$ is the frequency of mode $m, U_{\infty}$ is the free-stream velocity, $M_{\infty}$ is the free-stream Mach number, $L$ is the cavity length, $\gamma$ is the ratio of specific heats of the employed gas, $\alpha$ represents a phase shift, and $\kappa_{\nu}$ is the convection velocity coefficient of the vortices in the shear layer. These constants have the values $1.4,0.25$ and 0.57 respectively. The acoustic field around ideal cavities is well studied as documented in Lawson and Barakos [4]. However, the accurate prediction of the cavity acoustics can only be achieved by experiments or CFD which are expensive and time consuming.

Time averaged quantities as SPL, OASPL or the mean flow are well known in cavity flow. However, the unsteady cavity flow physics including standing waves, mode switching, and modulation of the tonal amplitude are not captured by time averaging, and retained attention only recently [5]. Joint time-frequency methods like the wavelet transform [6] are able to dissect the temporal behaviour of the tones, and have proved that there is no strong non-linear coupling between modes [5]. In this work, joint time-frequency methods are extended to perform a joint space-time-frequency analysis of the pressure field, to validate the flow dynamics of the obtained CFD solutions.

To further understand cavity flows, recent works applied novel experimental techniques, like the pressure sensitive paint [7] that gives pressure fluctuations over all cavity surfaces. Nevertheless, the sound pressure level peaks at the shear layer, are still difficult to measure without intrusive techniques. The beamforming technique for cavity flow, was used by Long [8] to decompose the pressure signals in coherent, and uncorrelated noise using a 
linear microphone array on the cavity ceiling. The present work apply this technique to reconstruct the noise sources using a microphone array in the far-field, which may help researchers to capture the acoustic field far from the walls using a limited number of probes.

This paper is organised as follows. First the CFD methodology, the postprocessing techniques, and the geometry are described. Then the employed CFD method is validated, and used with the beamforming technique.

\section{CFD Methodology}

The Helicopter Multi-Block (HMB3) [9] code is used for the present work. HMB3 solves the Unsteady Reynolds (Favre) Averaged Navier-Stokes (URANS) equations in integral form using the arbitrary Lagrangian-Eulerian (ALE) formulation, first proposed by Hirt et al. [10], for time-dependent domains, which may include moving boundaries. The Navier-Stokes equations are discretised using a cell-centred finite volume approach on a multi-block grid. The spatial discretisation of these equations leads to a set of ordinary differential equations in time,

$$
\frac{d}{d t}\left(\mathbf{w}_{i, j, k} V_{i, j, k}\right)=-\mathbf{R}_{i, j, k}(\mathbf{w})
$$

where $i, j, k$ represent the cell index, $\mathbf{w}$ and $\mathbf{R}$ are the vector of conservative variables and flux residual respectively and $V_{i, j, k}$ is the volume of the cell $i, j, k$. To evaluate the convective fluxes, the Osher [11] and Roe [12] approximate Riemann solvers are used and the viscous terms are discretised using a second order central differencing spatial discretisation. The Monotone Upstream-centred Schemes for Conservation Laws (MUSCL) of Leer [13], is used to provide third order accuracy in space. The HMB3 solver uses the Albada limiter [14] that is activated in regions where large gradients are encountered due to shock waves, avoiding non-physical, spurious oscillations. An implicit dual-time stepping method is employed to performed the temporal integration, where the solution is marching in pseudo-time iterations to achieve fast convergence, which is solved using a first-order backward difference. The linearised system of equations is solved using the Generalised Conjugate Gradient method with a Block Incomplete Lower-Upper (BILU) factorisation as a pre-conditioner [15]. The implicit scheme requires a small CFL at the early iterations or some explicit iteration using the forward Euler or four stage Runge-Kutta (RK4) methods [16]. Multi-block structured 
meshes are used with HMB3, which allow an easy sharing of the calculation load for parallel execution. The structured multi-block hexa meshes are generated using the ICEM-Hexa ${ }^{T M}$ tool of ANSYS. An overset grid method is available in HMB3 [17], to allow relative motion between mesh components. The chimera method is based on composite grids, consisting of independently generated, overlapping non-matching sub-domains. Each of these subdomains are referred to as a Level and are sorted hierarchically, with higher levels having priority. The exchange of information between sub-domains is achieved through interpolation and by following the level hierarchy. The work presented in this paper uses Scale-Adaptive Simulation (SAS) [18, 19], that has been successfully validated for cavity flows with and without doors using the HMB flow solver [20].

\section{Flow Analysis Methods}

This section presents the techniques used to analyse the unsteady flow data. CFD flow-field files are written at specific instances in time, and flow "probes" at specific mesh points are sampled at every time step. The probe pressure signals are first analysed using the Power Spectral Density (PSD), and the Overall Sound-Pressure Level (OASPL) [21].

\subsection{Time Frequency Analysis - Morlet Wavelet Method}

The cavity flow is highly unsteady, and its dynamics must be understood to gain insight in its physics. The continuous Morlet wavelet transform is a method for time-frequency analysis [22], that reveals the temporal fluctuations of the different frequencies present in the flow. The wavelet transform $W_{\Psi}^{y}(f, t)$ is a convolution of the signal $s(t)^{\prime}=s(t)-\bar{s}$ with a scaled mother wavelet $\Psi(t)$ conserving the sign of the signals in time and frequency:

$$
W_{\Psi}^{y}(a, b)=\frac{1}{\sqrt{c_{\Psi}|a|}} \int_{-\infty}^{\infty} s^{\prime}(t) \Psi\left(\frac{t-b}{a}\right) d t .
$$

In the above equation, $a$ is called the dilatation or the scale, $b$ the translation parameter, $c_{\Psi}=\sqrt{\pi / \beta}$ and $\beta=\omega_{0}^{2}$. The dilatation $a$ is related to the frequency $f$ of the wavelet, the translation parameter $b$ is related to the time shift $t$ of the wavelet. The mother, or Gabor wavelet $\Psi(t)$ is given by :

$$
\Psi(t)=e^{\frac{-\beta t^{2}}{2}} e^{j \omega t}
$$


Band Integrated Wavelets (BIW) plots show the energy content within a particular frequency range and is calculated using the following equation:

$$
B I W(t)=\int_{f_{1}}^{f_{2}} W_{\Psi}^{y}(f, t)^{2}
$$

where $f_{1}$ and $f_{2}$ are the lower and upper limits of the desired frequency range. The wavelets in decibel are given by:

$$
W_{d B}(f, t)=20 \mathrm{LOG}_{10}\left[\frac{W_{\Psi}^{y}(f, t)^{2}}{p_{\text {ref }}}\right]
$$

with $p_{\text {ref }}$ is the international standard for the minimum audible sound, which has the value of $2.10^{-5} \mathrm{~Pa}$ [23].

The wavelet envelop is the amplitude of the frequency in time, and is determined using the maximum of the absolute value of the wavelet transform over windows equal to half of a period of the frequency.

\subsection{Noise Source Analysis: Beamforming}

Cavity flows are characterised by high levels of noise. Typically, single microphone measurements, cannot distinguish between pressure contributions from different sources. Measurements from an acoustic array, instead, allow to determine the location of the acoustic sources, by means of a combination of the individual microphone signals [24]. This technique is useful for wind tunnel testing as it is not possible to measure the pressure at every point of the flow-field. The output of the beamforming algorithm is a noise map, which shows the power detected at each scanned grid point in terms of decibels below the peak power. Given an array with $M$ microphones, the waveform output of the $m$-th sensor will differ from the reference sensor of the array by a time delay. Therefore, the beamformer waveform output $z(t)$ can be written as the weighted sum of the sensor waveform outputs:

$$
z(t) \triangleq \sum_{m=0}^{M-1} y_{m}\left(t-\Delta_{m}\right),
$$

where $y_{m}(t)$ is the signal of the $m$-th microphone of the array, and $\Delta_{m}$ the time delay. The time delay is defined as $\Delta_{m} \triangleq \frac{r_{m}-r_{0}}{c}$, where $r_{m}$ is the distance from the assumed source position $\overrightarrow{x_{0}}$ and the $m$-th sensor and $c$ is the speed 
of sound. The conventional beamforming computes the output using the frequency representation of $z(t)$ obtained by a Fourier transformation of the microphones signals.

$$
Z(\omega)=\mathcal{F}\left\{\sum_{m=0}^{M-1} y_{m}\left(t-\Delta_{m}\right)\right\}=\sum_{m=0}^{M-1} Y_{\omega} e^{-j \Delta_{m} \omega} .
$$

If we define as $Y\left(\omega_{k}\right)$ an $M \times 1$ vector of complex numbers containing the signal amplitude and phase, at a frequency $\omega_{k}$, for each array sensor, and $e\left(\omega_{k}\right)$ as the steering $M \times 1$ vector containing the weight and phase delay information of the sensors for an assumed source location $\overrightarrow{x_{0}}$, then we can write

$$
Z\left(\omega_{k}\right)=e\left(\omega_{k}\right)^{\dagger} Y\left(\omega_{k}\right)=e_{k}^{\dagger} Y_{k},
$$

where $\mathrm{k}$ is the $k$-th frequency bin we can detect in the digital signal processing and ${ }^{\dagger}$ the Hermitian transpose operator.

The power detected at the $k$-th frequency bin is defined as

$$
P_{k} \triangleq\left|Z_{k}\right|^{2}=Z_{k} Z_{k}^{*}
$$

Therefore, using the definitions introduced before:

$$
P_{k}=e_{k}^{\dagger}\left(Y_{k} Y_{k}^{\dagger}\right) e_{k}=e_{k}^{\dagger} R e_{k}
$$

where $R$ is the cross spectral matrix. The results are characterised by the main lobe width, that has to be as narrow as possible, and side lobes at relatively lower levels that have to be as low as possible. This paper uses the Multiple Signal Classification (MUSIC) Algorithm to compute $P_{k}$ [25]. This is known to improve the response characteristics over linear techniques.

\subsubsection{Noise Propagation Model}

A sound wave around a transonic cavity flow travels in a non-uniform flowfield and has a speed close to the free-stream velocity. Consequently, the path to go from a source to a microphone is not a straight line. To use the beamforming algorithm, the distance between a source and a microphone has to take into account the path of the noise, and the noise propagation model is defined as follow. It is assumed that the noise sources radiate uniformly around them. The sound wave emitted in the direction of the microphone 
with an velocity $V_{0}$ of norm equal to the speed of sound $c$ is considered. The trajectory of the wave is computed taking into account the transport by the flow-field. The length of the trajectory $d_{\text {travel }}$ is the distance to go from the source to the microphone, and $c_{\text {travel }}$ the mean velocity along the trajectory. Then, the initial velocity of the sound wave $V_{0}$ is changed taking into account the offset distance $d_{\text {off }}$ between the trajectory and the microphone:

$$
V_{0}=V_{0}-d_{o f f} \frac{c_{\text {travel }}}{d_{\text {travel }}}
$$

The initial velocity is normalised to a norm $c$ and a new wave is launched with the new initial velocity. This process is repeated until a trajectory reaches the microphone. Furthermore, the wave does not have a constant velocity along the trajectory, therefore, the equivalent distance $r_{m}$ along the trajectory at a constant velocity $c$ is given by:

$$
r_{m}=d_{\text {travel }} \frac{c}{c_{\text {travel }}}
$$

This beamforming algorithm has been validated on a simple case of two speakers in front of an array of 40 microphones without free-stream. The microphone signals were sampled at $48 \mathrm{kHz}$ and recorded during 3 seconds. Figure 1 shows the microphone array in white dots and the beamforming result for the frequency of $1800 \mathrm{~Hz}$ generated by the speakers. The space is scanned for sources in a cube of $1 \mathrm{~m}$ side around the speakers. The speaker's positions are correctly found at $0.51 \mathrm{~m}$ in front of the microphone array. However, the lobes shown in the figure 1 are large in the sound propagation direction. More microphones distributed along the sound propagation direction are needed to reduce the lobe size.

\section{Geometric and Computational Model}

The simulations are carried out for the M219 cavity [26]. M219 has a length to depth ratio of 5 , a width to depth ratio of 1 , and a length of $0.51 \mathrm{~m}$. The experiments were carried out by Nightingale et al.[26] at a Mach Number of 0.85 , and a Reynolds $R e_{L}$ based on the cavity length of 6.5 million. Two cavities are computed, one with doors attached at its sides at an angle of 90 degrees (Figure 2), and an other with no doors. Experimental data were obtained using Kulite ${ }^{T M}$ pressure transducers at the cavity ceiling. Three 


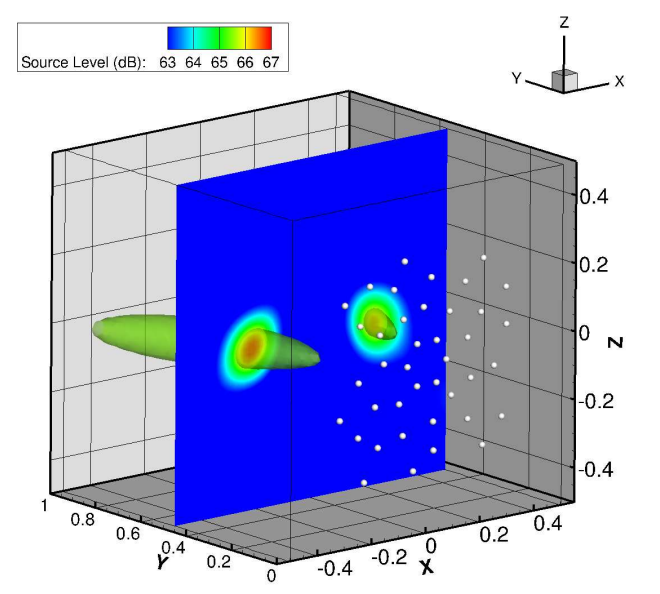

Figure 1: Validation of the beamforming analysis.

grid densities of 13, 22 and 34 million points are compared to the experimental data for the cavity with doors. The computations were performed using adimensional time-step of 0.01 , and are presented in table 1 .

\begin{tabular}{lcc}
\hline $\begin{array}{l}\text { Door } \\
\text { Angle }(\mathrm{deg})\end{array}$ & $\begin{array}{c}\text { Grid size } \\
\left(10^{6} \text { cells }\right)\end{array}$ & $\begin{array}{c}\text { Cavity Travel } \\
\text { Times }\end{array}$ \\
\hline 90 & 13.2 & 25 \\
90 & 22.3 & 25 \\
90 & 33.9 & 30 \\
No doors & 23.0 & 30 \\
\hline
\end{tabular}

Table 1: Details of the configurations computed with SAS and M219 cavity.

This paper uses different microphone arrays to show the effect of their position, density, and shape with the beamforming technique (Table 2). Two array shapes that give accurate source identification for far-field noise are tested [27]: the multi-spiral design (Figure 3a), composed of spirals equally rotated about the origin, and the Dougherty log-spiral design (Figure 3b), built with microphones equally spaced along a logarithmic spiral. The multispiral design is tested with 16, 21, 31, 61, and 101 microphones, and for different vertical positions. The arrays are centred on the aft wall of the cavity to take into account the flow-field that moves the focus point of the 


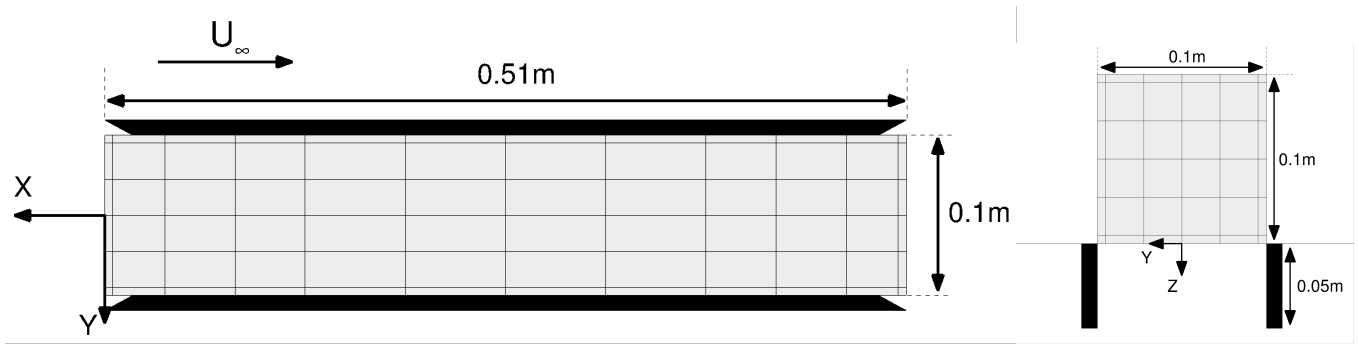

(a) Bottom view

(b) Downstream view

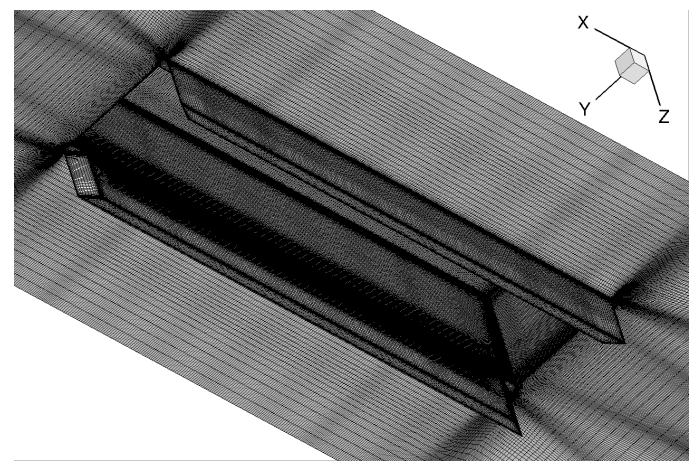

(c) Surface mesh

Figure 2: Schematic view of the M219 cavity with doors. Cavity in light grey and doors in black.

\begin{tabular}{lcc}
\hline Shape & Z/L & Nb microphone \\
\hline Multispiral & 0.6 & 31 \\
Multispiral & 0.9 & 31 \\
Multispiral & 1.2 & 31 \\
Multispiral & 1.5 & 16 \\
Multispiral & 1.5 & 21 \\
Multispiral & 1.5 & 31 \\
Multispiral & 1.5 & 61 \\
Multispiral & 1.5 & 101 \\
Dougherty & 1.5 & 31 \\
\hline
\end{tabular}

Table 2: Details of the microphone arrays 
array upstream. Those arrays could be fitted within the DERA Bedford wind-tunnel where this cavity was experimentally tested, where the section was $2.44 \mathrm{~m}$ by $2.74 \mathrm{~m}$ and where the cavity plate was $1 \mathrm{~m}$ away from the ceiling.

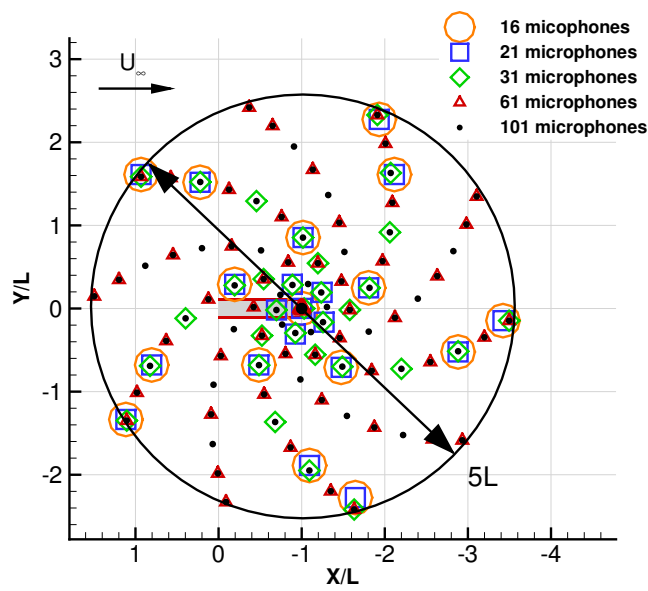

(a) Multi-spiral design

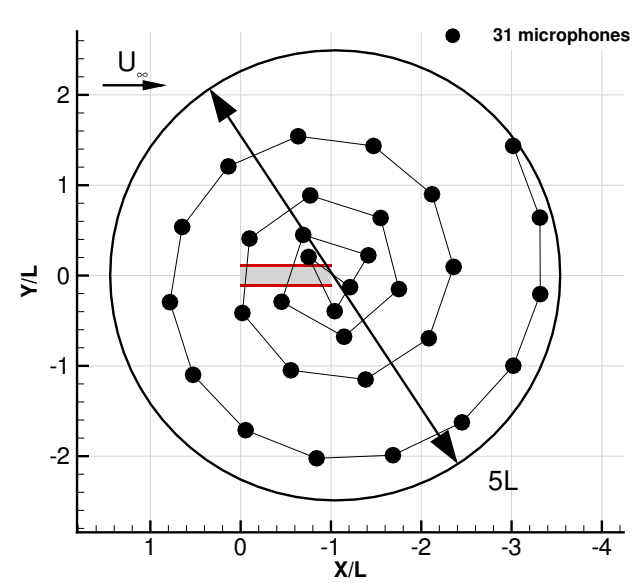

(b) Dougherty Design

Figure 3: Schematic view of the microphone arrays.

\section{Validation of the CFD Method}

\subsection{Averaged Pressure Validation}

Figure 4 shows the SPL comparison between CFD and experiments at three points at $5 \% \mathrm{~L}, 45 \% \mathrm{~L}$, and $95 \% \mathrm{~L}$ on the cavity mid-span, and on the ceiling, for the M219 cavity with doors. Since the CFD simulations are run for a typical length of 25 travel times, and the experimental data span 1900 travel times, the comparison is carried out as follows. The experiment is divided in windows of 25 travel times, and the result leads to the envelop shown with the SPL. The SPL shows better agreement with the test data when the fine grid is used, capturing both tonal and broadband noise.

The time averaged $C_{P}$ (Figure 5a) at the ceiling, and at the mid-span of the cavity, shows the grid convergence, with negligible changes between the different grid densities. The OASPL, on figure 5b is shown with error bars, 


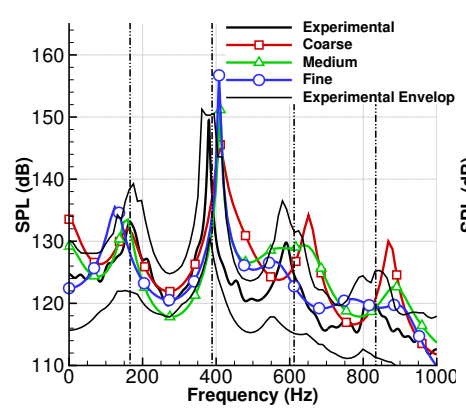

(a) $\mathrm{X} / \mathrm{L}=0.05$

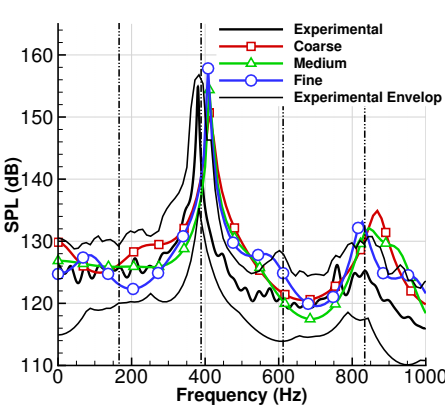

(b) $\mathrm{X} / \mathrm{L}=0.45$

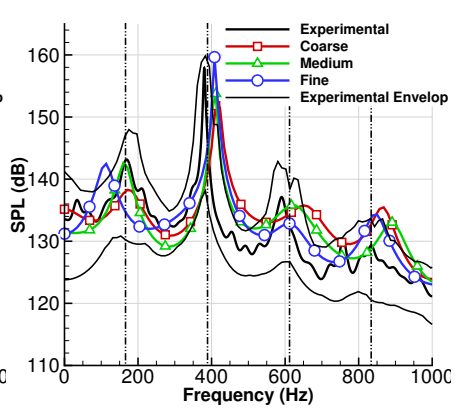

(c) $\mathrm{X} / \mathrm{L}=0.95$

Figure 4: SPL at the M219 cavity with door at the ceiling mid-span for CFD and experimental signals. Vertical black lines represent the Rossiter modes.

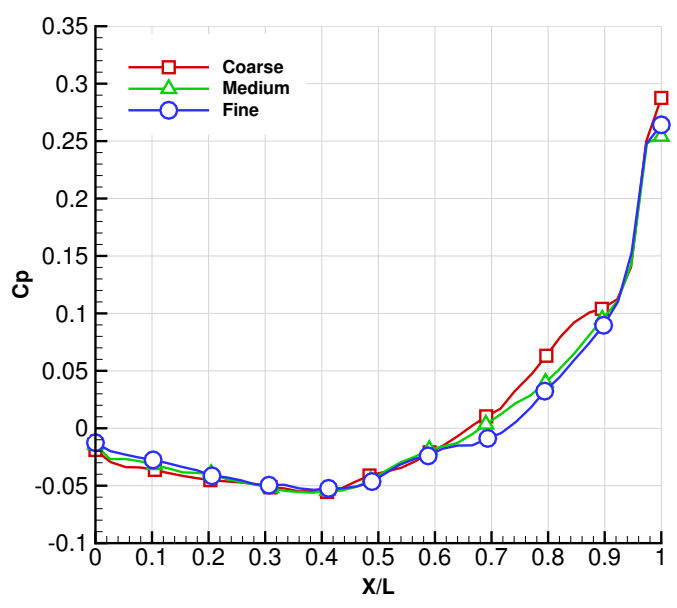

(a) Mean Cp

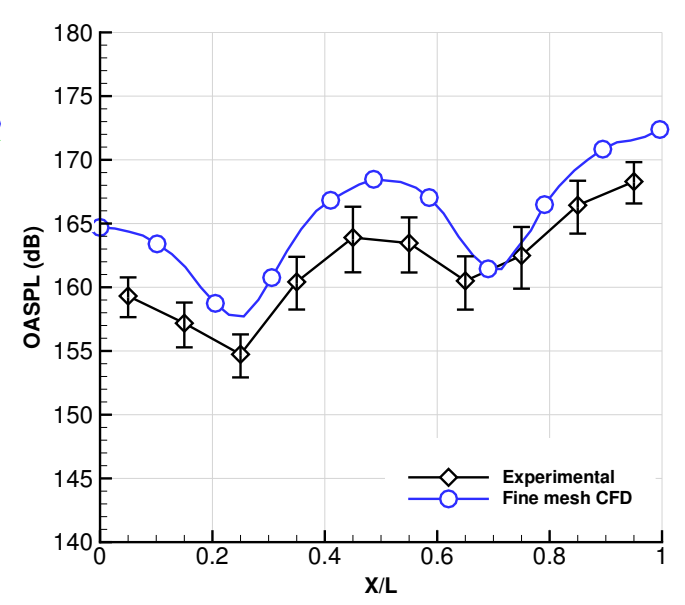

(b) OASPL

Figure 5: OASPL, and mean $C_{P}$ along the M219 cavity ceiling mid-span.

computed as for figure 4. The second Rossiter mode is dominant, with a W shape of the OASPL, as captured by the CFD and the experiments [28].

The CFD is compared with the experiments for the cavity without doors on figure 6. Overall, the CFD captured well the differences between the door and no door configurations, including the strong increase of the second cavity mode with the doors, suggesting that SAS is suitable method for this flow.

The time averaged stream-wise velocity is compared in figure 7 between 


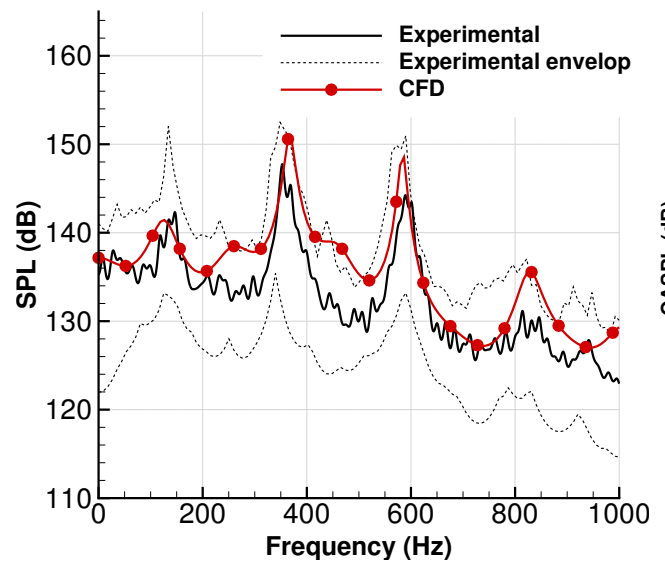

(a) SPL

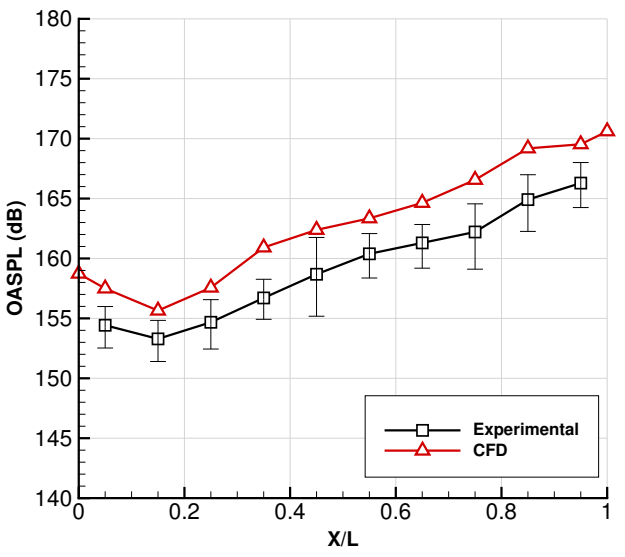

(b) OASPL

Figure 6: Noise along the no doors M219 cavity ceiling mid-span.

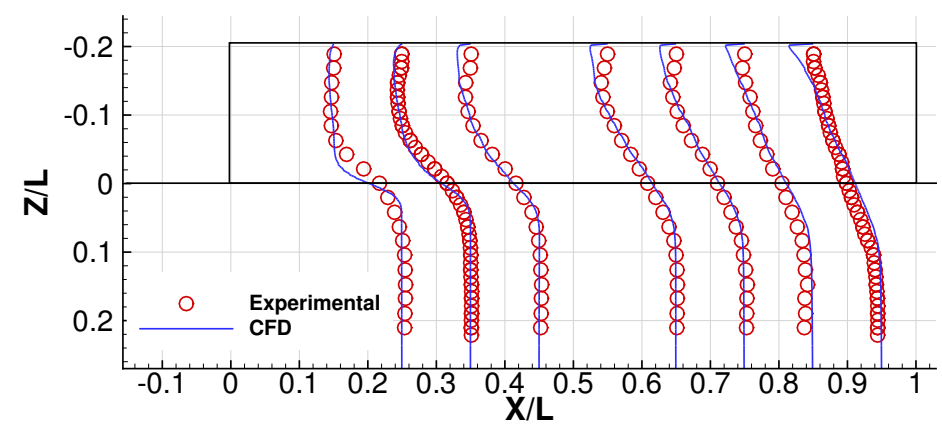

Figure 7: Time averaged stream-wise velocity at the mid-span of the cavity along vertical lines.

CFD and PIV experiments [29] for the M219 cavity without doors. The CFD results agree well with the experiments, showing the development of the shear layer along the cavity length.

\subsection{Spatio-Temporal Pressure Validation}

The wavelet transform is used to perform spatio-temporal validation of the CFD signals. The pressure probes are analysed, and the Banded Integral Wavelet (BIW) is represented on figure 8 for 25 travel times, along the ceiling centre line. The scalogram is integrated in windows of $20 \mathrm{~Hz}$ centred on the 
first, and second cavity modes. There is a fair agreement of the CFD with the experiments, showing standing waves, characterised by nodes (minima of amplitude), and antinodes (maxima of amplitude). Furthermore, there is phase opposition between neighbouring antinodes. This behaviour is also seen for higher frequency modes, as also pointed out by Rossiter [1] in the construction of his model for the flow flow dynamics and acoustics.

The BIW envelop is shown figure 9. The CFD signal agrees with the

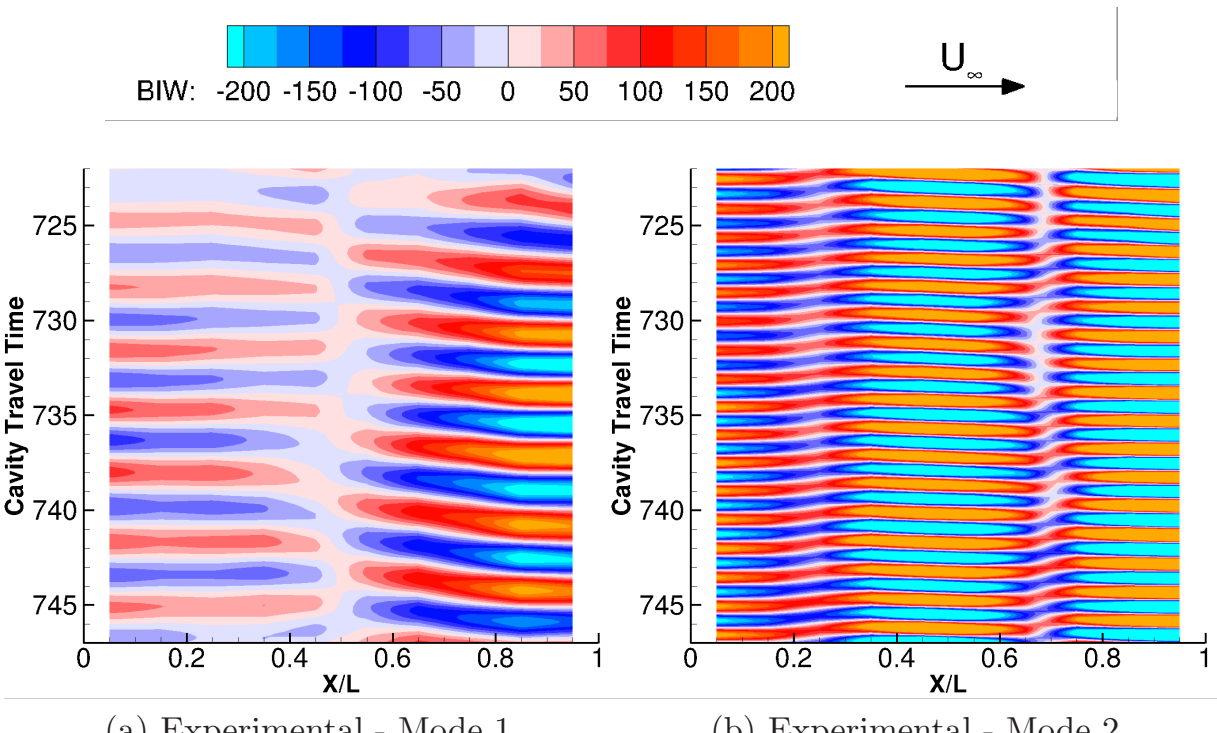

(a) Experimental - Mode 1

(b) Experimental - Mode 2

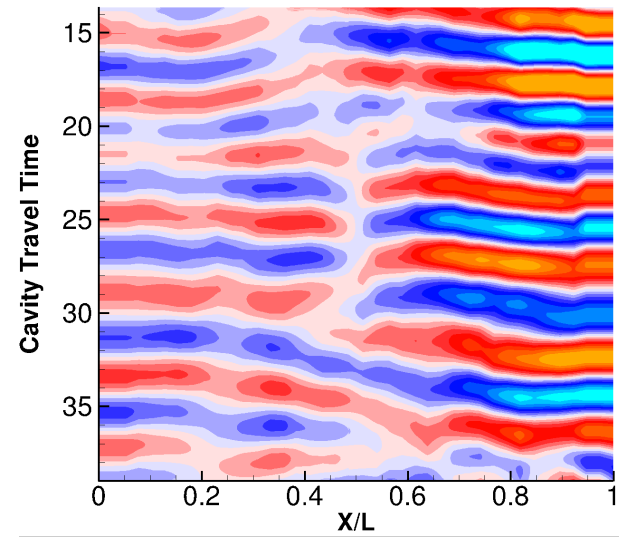

(c) CFD - Mode 1

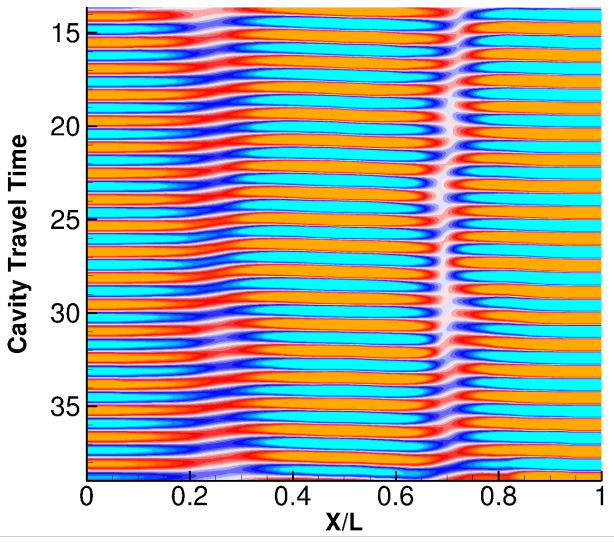

(d) CFD - Mode 2

Figure 8: BIW at the cavity ceiling centre-line for modes 1 and 2. 
experiments showing the characteristic shape of the first mode, with two antinodes, one at the front, and one at the aft wall. The second mode shows the W shape seen on the OASPL. The modal amplitude is not constant over time, with global fluctuations all along the cavity. For example, the second CFD mode (Figure 9d) shows three maxima at travel times of 19, 27, and 35. Those amplitude oscillations, denoted as mode amplitude modulation, were also described in experimental works [5]. The SAS model shows good

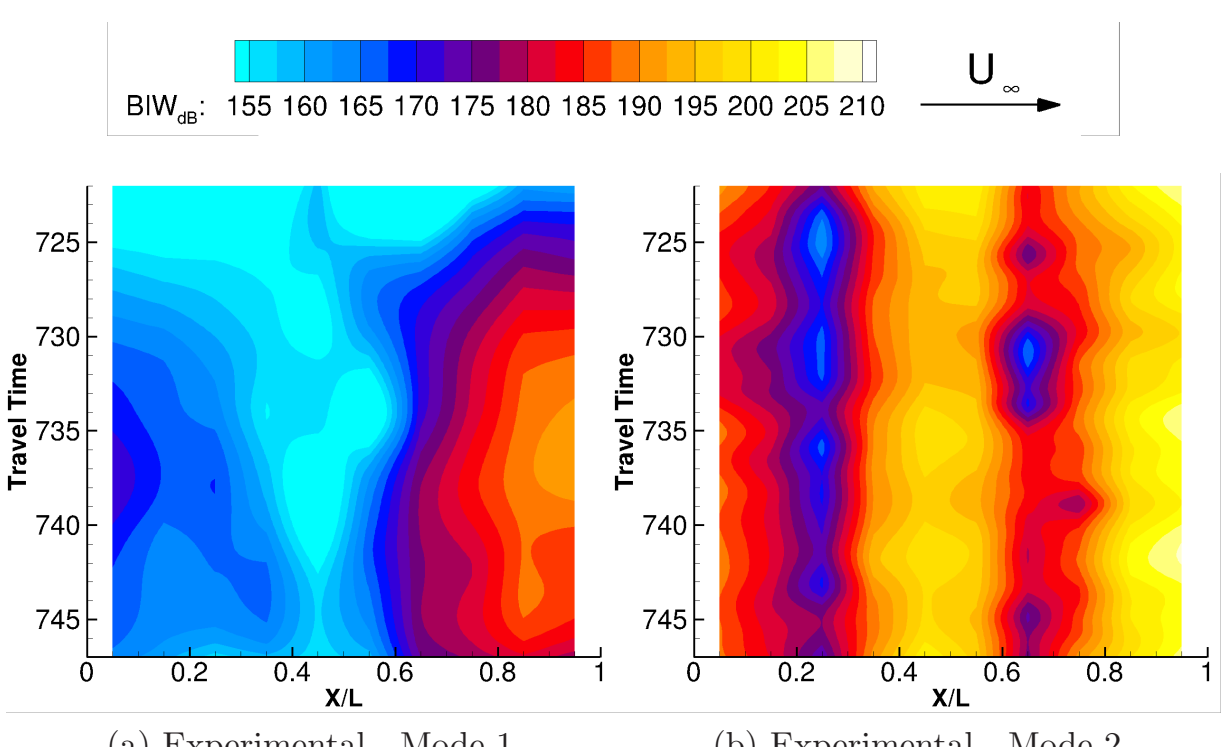

(a) Experimental - Mode 1

(b) Experimental - Mode 2

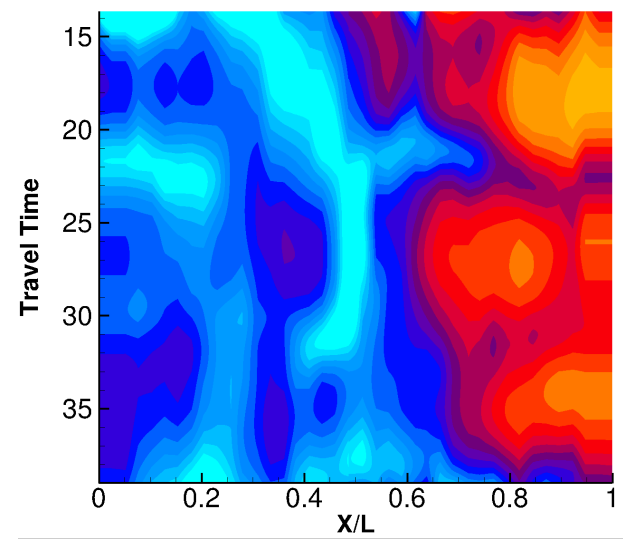

(c) CFD - Mode 1

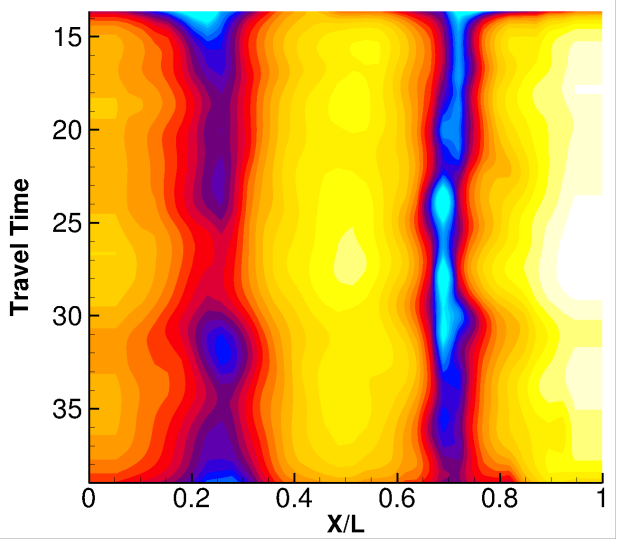

(d) CFD - Mode 2

Figure 9: BIW amplitude at the cavity ceiling centre-line for modes 1 and 2. 
agreement with experiments for the transonic cavity pressure field on average, and in terms of spatio-temporal components.

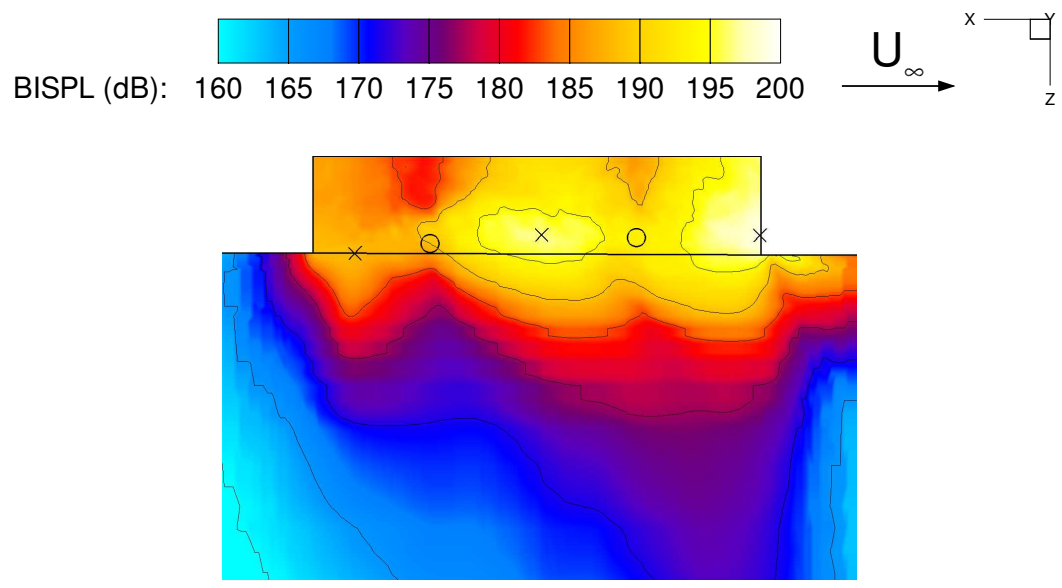

(a) 10 to $1300 \mathrm{~Hz}$

BISPL (dB): $\begin{array}{llllllllllll}130 & 135 & 140 & 145 & 150 & 155 & 160 & 165 & 170 & 175 & \mathrm{U}_{\infty}\end{array}$

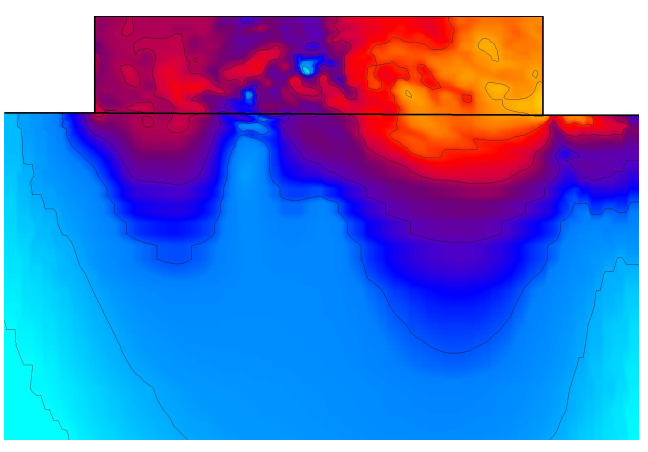

(b) Mode 1

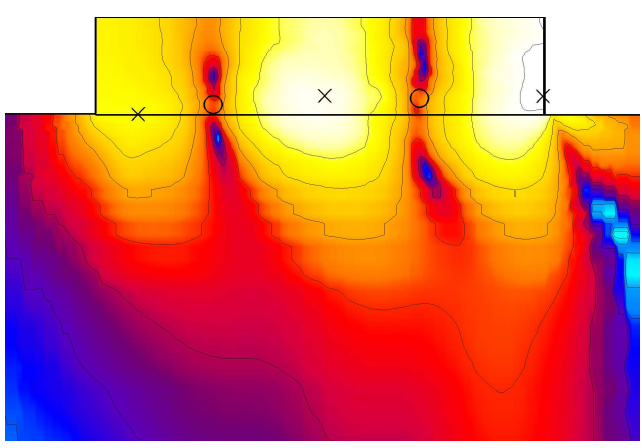

(c) Mode 2

Figure 10: BISPL at the mid-span of the cavity over a large frequency band (a), and centred on the two first cavity modes in windows of $10 \mathrm{~Hz}$ (b)-(c). 


\section{Results and Discussion}

\subsection{Description of the Noise Field}

Using the full CFD flow-field of the M219 cavity with doors, the noise field is computed and shown in figure 10 at the mid-span of the cavity. Between 10 and $1300 \mathrm{~Hz}$ (Figure 10a), two main sources of noise are localised at the mid-length, and at the aft of the shear layer, due to the strong second cavity mode. The BISPL, is also integrated around cavity modes 1 and 2 in windows

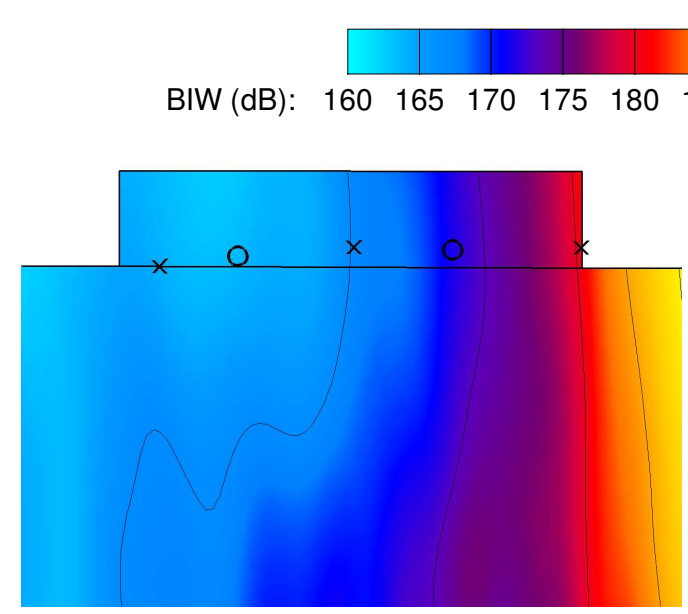

(a) No flow-field

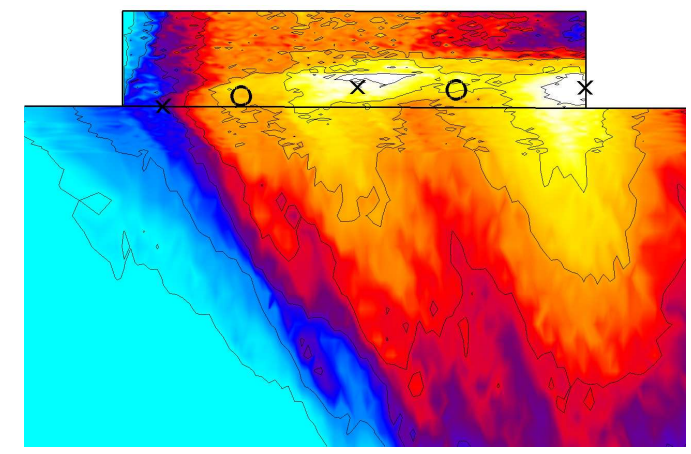

(b) Ideal flow-field

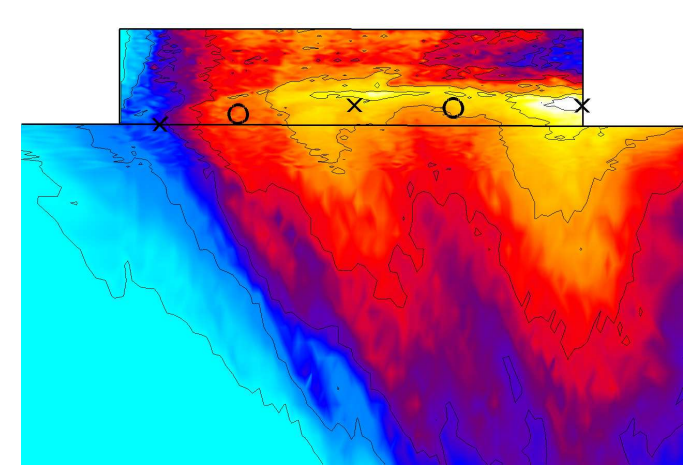

(c) Mean flow-field

Figure 11: BIW at the mid-span of the cavity over a large frequency band between 10 and $1300 \mathrm{~Hz}$ for different noise propagation model. Multi-spiral array with 31 microphones, placed at $\mathrm{Z} / \mathrm{L}=1.5$. 
of $10 \mathrm{~Hz}$ width (Figures 10b and 10c). All modes show alternatively high and low noise levels along the cavity length, corresponding to the nodes, and the antinodes of standing waves. The nodes of the dominant second mode are shown with circles, and the antinodes with crosses, in figures 10 to 15. The cavity modes are mainly produced along the shear layer, as seen by the peaks of the different modes close to the black line at $\mathrm{Z}=0$.

Experiments are limited to pressure probes placed on the wall, so they miss some important characteristics of the noise field as described above. The beamforming is applied to the CFD results, with the objective to obtain results comparable to the BISPL computed using all the CFD points on the mesh. The static pressure is considered at the microphone as the hydrodynamic fluctuations are negligible far from the cavity. The noise is reconstructed on a grid of 7290 points at the mid-span of the cavity using the multi-spiral array with 31 microphones, placed at $\mathrm{Z} / \mathrm{L}=1.5$. Figure 11 shows the effect of the level of accuracy of the flow-field used to transport the sound waves. First, a zero velocity flow-field is assumed, and the pressure waves propagate along straight lines from the scanned points to the microphones at the speed of the sound (Figure 11a). The noise in this case is not correctly localised, with a peak downstream of the cavity. Assuming pressure waves travelling in an ideal flow-field (Figure 11b) with zero velocity in the

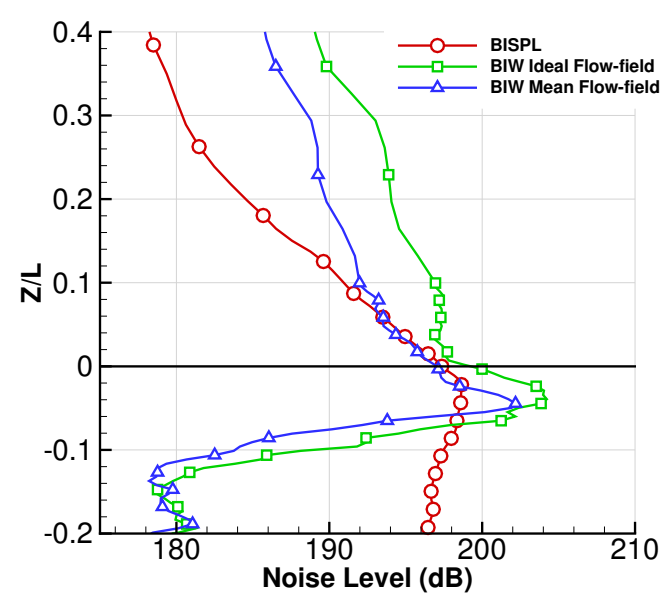

(a) $\mathrm{X} / \mathrm{L}=-0.95$

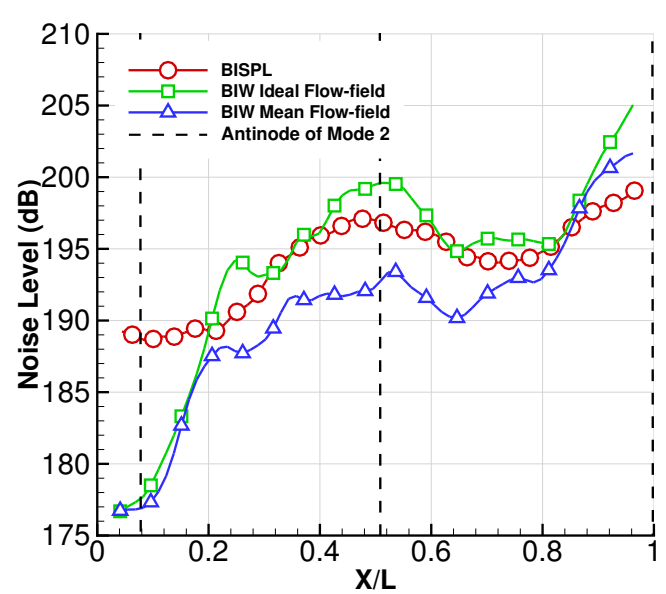

(b) $\mathrm{Z} / \mathrm{L}=-0.04$

Figure 12: Comparison between OASPL and BIW along lines. Multi-spiral array with 31 microphones, placed at $\mathrm{Z} / \mathrm{L}=1.5$. 
cavity, and free-stream outside, the noise is more accurately localised around the shear layer, mainly at the second, and at the third antinodes of the second cavity mode (Second and third crosses in figure 11b). Nevertheless, the sources of noise are surrounded by large lobes along the vertical direction.

Employing the CFD mean flow-field to transport the waves(Figure 11c), the sources of noise are localised at the same axial position compared to the ideal flow-field case (Figure 12b). However, the lobes are smaller for the mean flow-field case, as the thickness of the shear layer, is taken into account, giving a more precise vertical localisation of the noise sources (Figure 12a). The shape of the noise field bellow the cavity is also correctly reconstructed, with higher levels of noise at the second half of the cavity. The source of noise

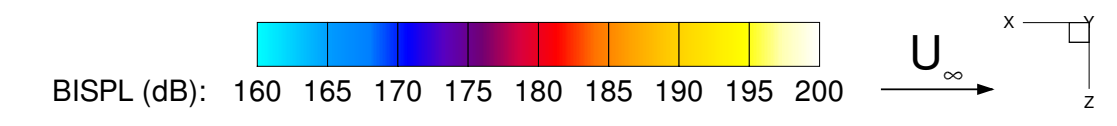

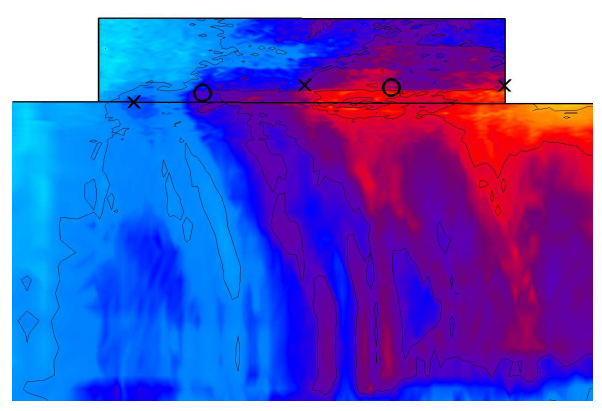

(a) $\mathrm{Z} / \mathrm{L}=0.60$

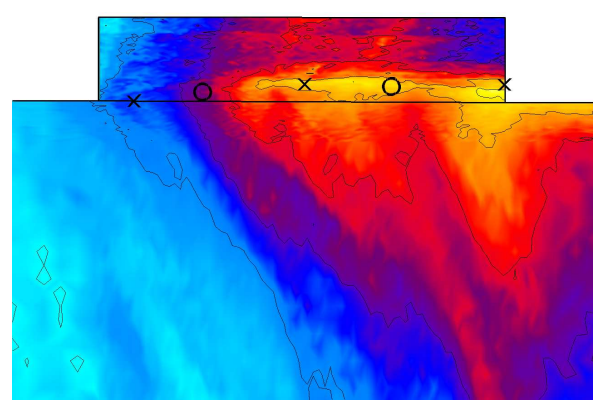

(c) $\mathrm{Z} / \mathrm{L}=1.20$

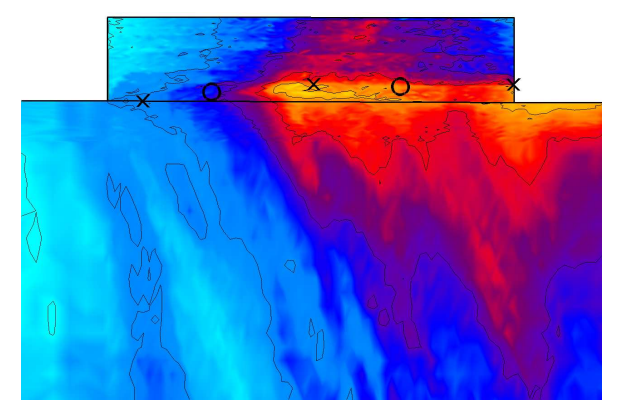

(b) $\mathrm{Z} / \mathrm{L}=0.90$

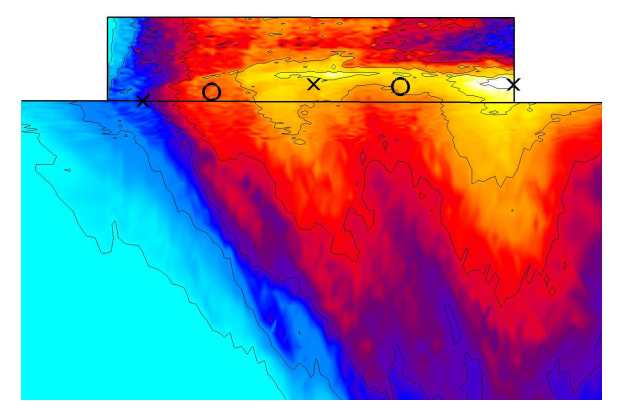

(d) $\mathrm{Z} / \mathrm{L}=1.50$

Figure 13: BIW at the mid-span of the cavity over a large frequency band between 10 and $1300 \mathrm{~Hz}$ for different multi-spiral array positions. Multi-spiral array with 31 microphones. 
at the front of the cavity is weaker than for the other parts of the cavity, and is hidden by the lobes of the stronger noise sources. In the following, the mean flow is used for all beamforming results.

Figure 13 shows the influence of the vertical position of the multi-spiral array of 31 probes. The array closer to the cavity at $\mathrm{Z} / \mathrm{L}=0.6$ (Figure 13a), did not capture the sources at the shear layer, and the noise field close to the microphone array was not correctly reconstructed. Moving the array farther from the cavity, improves the reconstruction. The two main noise sources at $50 \% \mathrm{~L}$ and at $100 \% \mathrm{~L}$ of the shear layer are more visible, and the spread of the noise inside the cavity compares better with the BISPL. When the array is too close to the cavity, the near field influences the resulting reconstruction, leading to errors [30].

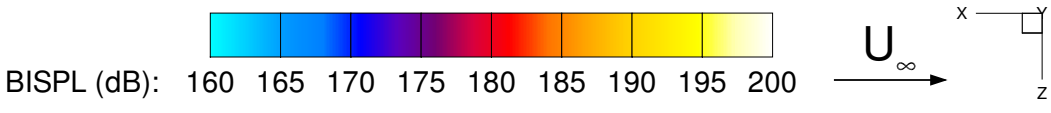

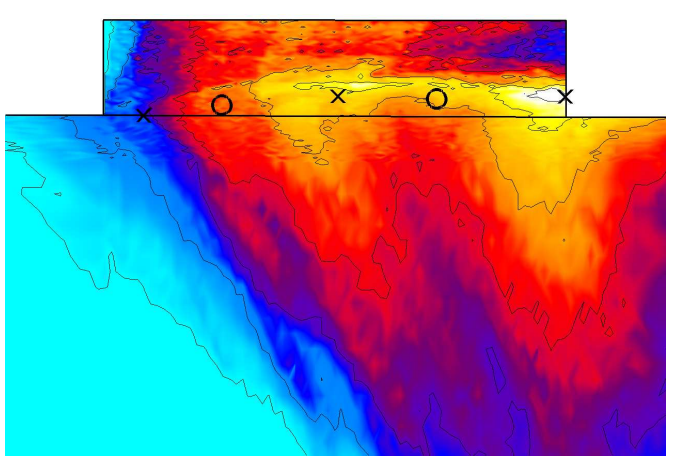

(a) Multi-spiral array

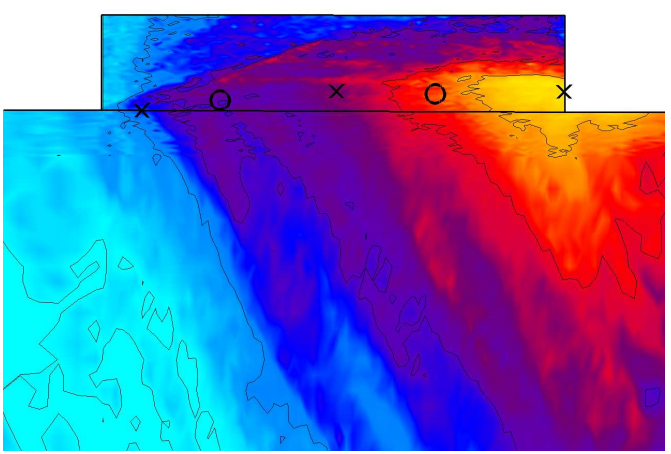

(b) Dougherty array

Figure 14: BIW at the mid-span of the cavity over a large frequency band between 10 and $1300 \mathrm{~Hz}$ for different array shapes. Array of 31 microphones placed at $\mathrm{Z} / \mathrm{D}=1.5$.

Figure 14 shows the beamforming for the multi-spiral and the Dougherty arrays of 31 microphones, placed at 1.5L from the cavity. The Dougherty design reconstruction did not capture the noise source at $50 \% \mathrm{~L}$ of the shear layer, and globally is less accurate than the multi-spiral array reconstruction. This may be the consequence of the lower density of microphones close to the perimeter of the Dougherty array, which reduces its bandwidth.

Figure 15 shows that the accuracy of the beamforming depends on the 


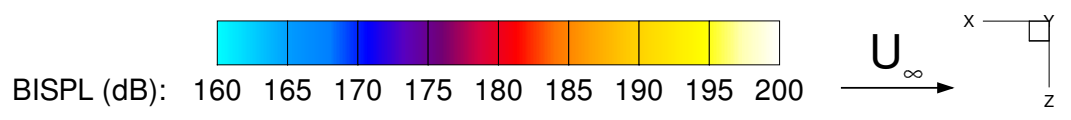

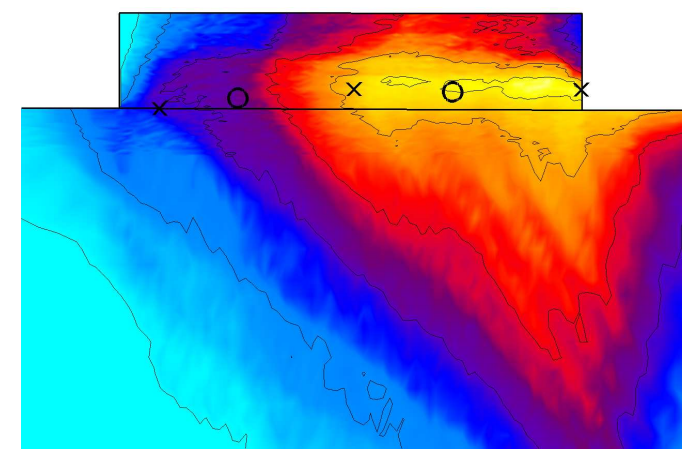

(a) 101 microphones

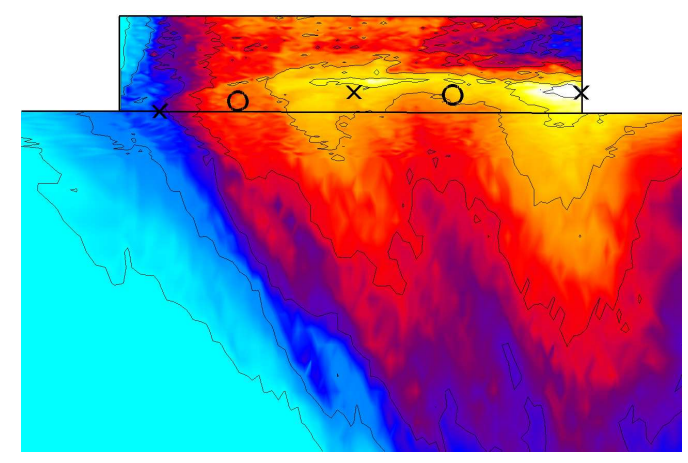

(c) 31 microphones

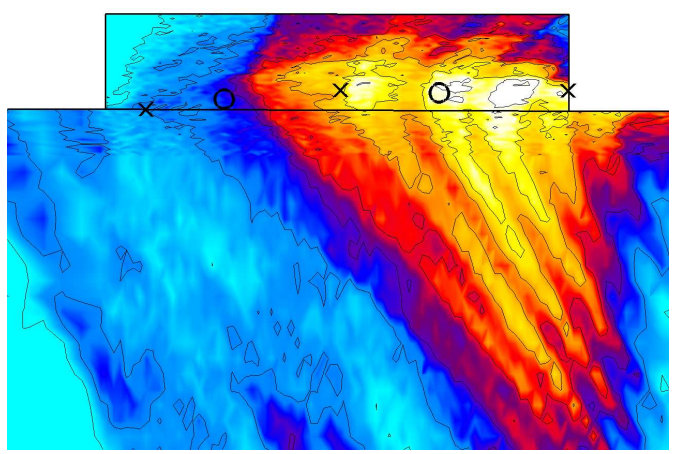

(b) 61 microphones

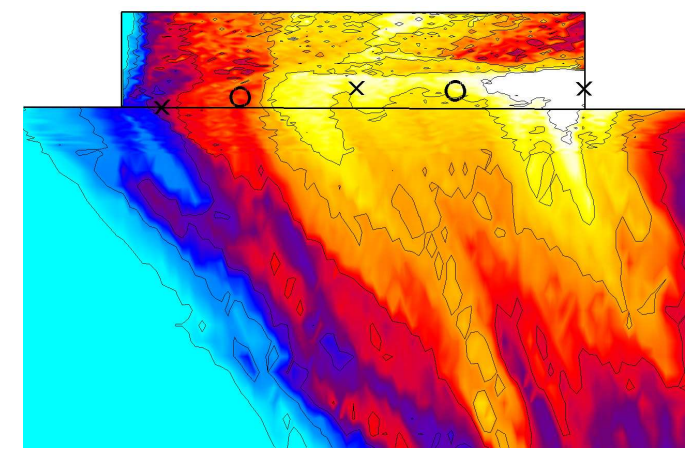

(d) 21 microphones

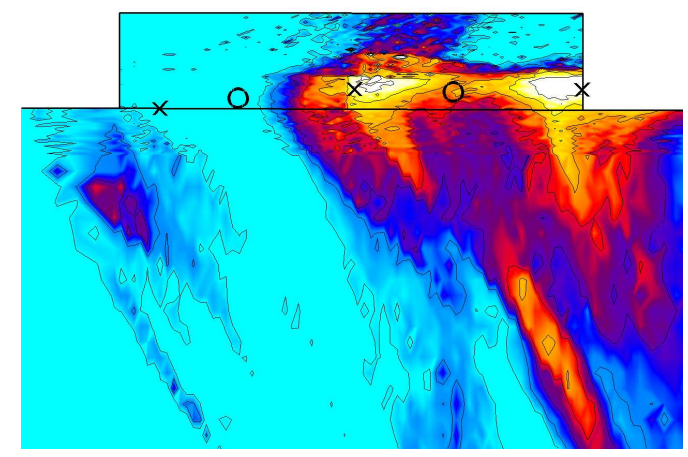

(e) 16 microphones

Figure 15: BIW at the mid-span of the cavity over a large frequency band between 10 and $1300 \mathrm{~Hz}$ for different array density. Multi-spiral array placed at $\mathrm{Z} / \mathrm{L}=1.5$. 
number of microphones. When a large number of microphones are used, the strong broadband noise is amplified when summed over all microphones, and hides the main sources (Figures 15a and 15b). On the other hand, the results with a small number of microphones are influenced by background noise increasing the lobe size with 21 microphones (Figure 15d), and leading to non physical noise sources bellow the cavity for 16 microphones (Figure 15e). The best configuration tested here had 31 microphones (Figure 15c).

Figure 16 compares the BISPL and the beamforming for the M219 cavity without doors. Both the beamforming, and the BISPL, show the reduction of the noise source amplitude at the mid-length of the cavity, caused by a weaker second cavity mode, without the doors.

This first attempt to apply the beamforming to localise noise sources within cavity flows is encouraging, and suggests that if applied to wind tunnels, more physics could be observed from cavity flows, and more data could be obtained for CFD validation.

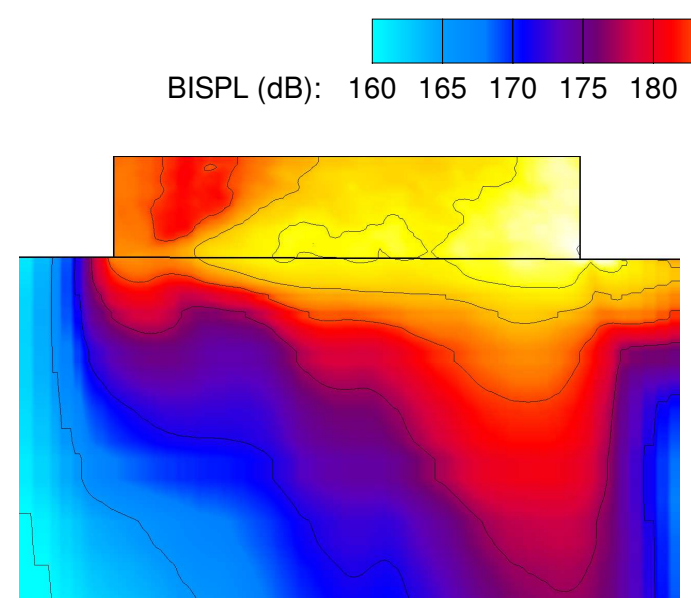

(a) BISPL
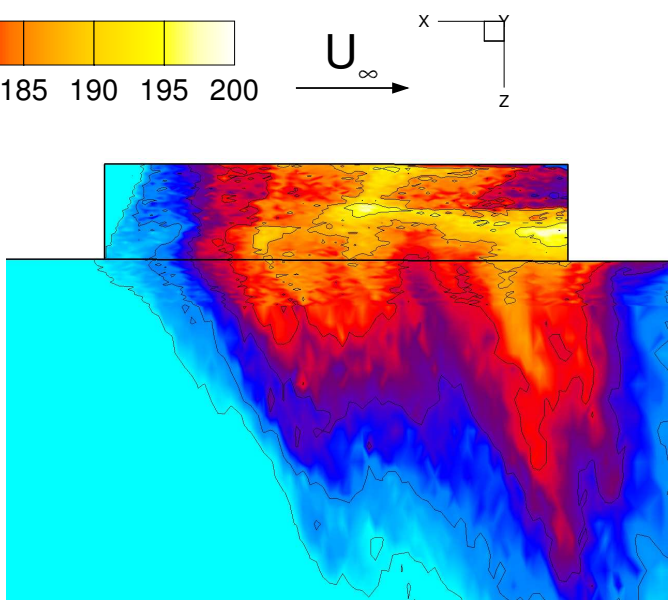

(b) Beamforming

Figure 16: Noise field at the mid-span of the M219 cavity without doors over a large frequency band between 10 and $1300 \mathrm{~Hz}$. Multi-spiral array of 31 microphones placed at $\mathrm{Z} / \mathrm{D}=1.5$. 


\section{Conclusions}

This work focused on the analysis of transonic cavity flow data to give more insight on its physical mechanisms.

The beamforming proved to be capable to capture the noise field around the cavity using a limited number of probes, providing the mean CFD flowfield to compute the propagation of the noise. This technique could be used in wind tunnels, coupling microphone array measurement, and PIV data. This method provides meaningful results if the array is far enough from the cavity. In addition, the optimal number of microphones must be determined to capture the tonal sources of noise. A large number of microphone amplifies the broadband noise, and hides the tones, while a small number of microphones can not localise the main sources. The shape of the array also influences the results and should have a large density of microphone on its periphery. As a result, further cavity flows physics, and more data could be obtained for CFD validation.

Acknowledgments

The financial support of MBDA UK Ltd. is gratefully acknowledged. The use of the EPSRC funded ARCHIE-WeSt High Performance Computer (EPSRC grant no. EP/K000586/1) is also gratefully acknowledged

\section{References}

[1] Rossiter, J. E., "Wind Tunnel Experiments on the Flow Over Rectangular Cavities at Subsonic and Transonic Speeds," Technical Report 64037, Royal Aircraft Establishment, Bedford, UK, October 1964.

[2] Schmit, R., Grove, J., Semmelmayer, F., and Haverkamp, M., "Nonlinear feedback mechanisms inside a rectangular cavity," AIAA Journal, Vol. 52, No. 10, 2014, pp. 2127 - 2142, doi:10.2514/1.J052804.

[3] Heller, H., Holmes, D., and Covert, E., "Flow-Induced Pressure Oscillations In Shallow Cavities," Journal of Sound and Vibration, Vol. 18, No. 4, 1971, pp. 545 - 553, doi:10.1016/0022-460X(71)90105-2.

[4] Lawson, S. and Barakos, G., "Review of Numerical Simulations For High-Speed, Turbulent Cavity Flows," Progress in Aerospace Sciences, Vol. 47, No. 3, 2011, pp. 186 - 216, doi:10.1016/j.paerosci.2010.11.002. 
[5] Kegerise, M. A., Spina, E. F., Garg, S., and III, L. N. C., "Mode-switching and nonlinear effects in compressible flow over a cavity," Physics of Fluids, Vol. 16, No. 3, 2004, pp. 678-687, doi:10.1063/1.1643736.

[6] Wagner, J., Casper, K., Beresh, S., Arunajatesan, S., Henfling, J., Spillers, R., and Pruett, B., "Relationship between acoustic tones and flow structure in transonic cavity flow," Proceedings of the 45th AIAA Fluid Dynamics Conference, AIAA AVIATION Forum, Paper No. AIAA 2015-2937, American Institute of Aeronautics and Astronautics Inc, Dallas, TX, United States, 2015, doi:10.2514/6.2015-2937.

[7] Flaherty, W., Reedy, T., Elliott, G., Austin, J., Schmit, R., and Crafton, J., "Investigation of cavity flow using fast-response pressuresensitive paint," AIAA Journal, Vol. 52, No. 11, 2014, pp. 2462-2470, doi:10.2514/1.J052864.

[8] Long, D., "Spatial structure of cavity pressure fluctuations at transonic speeds," AIAA Journal, Vol. 44, No. 9, 2006, pp. 1983-1992, doi:10.2514/1.5236.

[9] Lawson, S. J., Steijl, R., Woodgate, M., and Barakos, G. N., "High performance computing for challenging problems in computational fluid dynamics," Progress in Aerospace Sciences, Vol. 52, No. 1, 2012, pp. 19 29, doi: 10.1016/j.paerosci.2012.03.004.

[10] Hirt, C. W., Amsten, A. A., and Cook, J. L., "An Arbitrary Lagrangian-Eulerian Computing Method for All Flow Speeds," Journal of Computational Physics, Vol. 14, No. 3, 1974, pp. 227-253, doi: 10.1006/jcph.1997.5702.

[11] Osher, S. and Chakravarthy, S., "Upwind Schemes and Boundary Conditions with Applications to Euler Equations in General Geometries," Journal of Computational Physics, Vol. 50, No. 3, 1983, pp. 447-481, doi:10.1016/0021-9991(83)90106-7.

[12] Roe, P., "Approximate Riemann Solvers, Parameter Vectors and Difference Schemes," Journal of Computational Physics, Vol. 43, No. 2, 1981, pp. 357-372, doi:10.1016/0021-9991(81)90128-5. 
[13] van Leer, B., "Towards the ultimate conservative difference scheme. V.A second-order sequel to Godunov's Method," Journal of Computational Physics, Vol. 32, No. 1, 1979, pp. 101-136, doi:10.1016/00219991(79)90145-1.

[14] van Albada, G., van Leer, B., and Roberts, W., A Comparative Study of Computational Methods in Cosmic Gas Dynamics, Upwind and HighResolution Schemes, edited by M. Y. Hussaini, B. van Leer, and J. Van Rosendale, Springer Berlin Heidelberg, Berlin, Heidelberg. Germany, 1997, pp. 95-103, doi:10.1007/978-3-642-60543-7_6.

[15] Axelsson, O., Iterative Solution Methods, Cambridge University Press, Cambridge, MA, United States, 1994, pp. 504-557, doi:10.1017/CBO9780511624100.

[16] Jameson, A., Schmidt, W., and Turkel, E., "Numerical Solutions of Euler Equations by Finite Volume Methods Using Runge-Kutta TimeStepping Schemes," Proceedings of the Fourteenth Fluid and Plasma Dynamic Conference, Paper No. 1981-1259, American Institute of Aeronautics and Astronautics Inc, Palo Alto, CA, United States, 1981, pp. 1-19, doi:10.2514/6.1981-1259.

[17] Jarkowski, M., Woodgate, M., Barakos, G., and Rokicki, J., "Towards Consistent Hybrid Overset Mesh Methods for Rotorcraft CFD," International Journal for Numerical Methods in Fluids, Vol. 74, No. 8, 2014, pp. 543-576, doi:10.1002/fld.3861.

[18] Menter, F. and Egorov, Y., "The Scale-Adaptive Simulation Method for Unsteady Turbulent Flow Predictions. Part 1: Theory and Model Description," Flow, Turbulence and Combustion, Vol. 85, No. 1, 2010, pp. 113-138, doi:10.1007/s10494-010-9264-5.

[19] Egorov, Y., Menter, F., Lechner, R., and Cokljat, D., "The ScaleAdaptive Simulation Method for Unsteady Turbulent Flow Predictions. Part 2: Application to Complex Flows," Flow, Turbulence and Combustion, Vol. 85, No. 1, 2010, pp. 139-165, doi:10.1007/s10494-010-9265-4.

[20] Babu, S., Zografakis, G., and Barakos, G., Evaluation of scale-adaptive simulations for transonic cavity flows, Progress in Hybrid RANSLES Modelling: Papers Contributed to the 5th Symposium on Hybrid 
RANS-LES Methods, 19-21 March 2014, College Station, AEM University, Texas, USA, edited by S. Girimaji, W. Haase, S.-H. Peng, and D. Schwamborn, Vol. 130, Springer International Publishing, 2015, pp. 433-444, doi:10.1007/978-3-319-15141-0_35.

[21] Babu, S., Loupy, G., Dehaeze, F., Barakos, G., and Taylor, N., "Aeroelastic simulations of stores in weapon bays using Detached-Eddy Simulation," Journal of Fluids and Structures, Vol. 66, October 2016, pp. 207228, doi:10.1016/j.jfluidstructs.2016.07.014.

[22] Bussow, R., "An algorithm for the continuous Morlet wavelet transform," Mechanical Systems and Signal Processing, Vol. 21, No. 8, 2007, pp. 2970-2979, doi:10.1016/j.ymssp.2007.06.001.

[23] Pierce, A., Acoustics: An Introduction to its Physical Principles and Applications, Woodbury, New York: Acoustical Society of America, 1989.

[24] Schmidt, R. O., "Multiple Emitter Location and Signal Parameter." IEEE Transactions on Antennas and Propagation, Vol. AP-34, No. 3, 1986, pp. 276-280, doi:10.1109/TAP.1986.1143830.

[25] Simley, E., Developement of an Acoustic Array for Wind Turbine Aeroacoustic Noise Analysis., Ph.D. thesis, University of Colorado, April 2010.

[26] Nightingale, D., Ross, J., and Foster, G., "Cavity Unsteady pressure measurements - Examples from Wind-Tunnel Tests," Tech. Rep. Version 3, Aerodynamics \& Aeromechanics Systems Group, QinetiQ, Bedford, UK, November 2005.

[27] Prime, Z. and Doolan, C., "A comparison of popular beamforming arrays," Proceedings of the Annual Conference of the Australian Acoustical Society 2013, Australian Acoustical Society, Victor Harbor, SA, Australia, 2013, pp. 151-157.

[28] Lee, B., "Effect of Captive Stores on Internal Weapons Bay Floor Pressure Distributions," Journal of Aircraft, Vol. 47, No. 2, March-April 2010, pp. 732-735, doi:10.2514/1.46684.

[29] Ross, J. A., "PIV Measurements of the flowfields in an Aerodynamically Deep Cavity," Tech. rep., Aerodynamics \& Aeromechanics Systems Group, QinetiQ, Bedford, UK, October 2002. 
[30] Ryan, J. G., "Criterion for the minimum source distance at which plane-wave beamforming can be applied," The Journal of the Acoustical Society of America, Vol. 104, No. 1, 1998, pp. 595-598, doi:10.1121/1.423289. 